\title{
Capillary discharge-driven metal vapor plasma waveguides
}

\author{
Y. Wang, B. M. Luther, M. Berrill, M. Marconi, F. Brizuela, and J. J. Rocca \\ NSF ERC for Extreme Ultraviolet Science and Technology, and Electrical and Computer Engineering Department, \\ Colorado State University, Fort Collins, Colorado 80523, USA \\ V. N. Shlyaptsev \\ Department of Applied Science, University of California Davis-Livermore, Livermore, California 94551, USA
}

(Received 1 November 2004; revised manuscript received 8 June 2005; published 26 August 2005)

\begin{abstract}
We report the generation of dense plasma waveguides containing a large concentration of silver ions by means of a fast ( $\sim 55 \mathrm{~ns}$ first half-cycle) microcapillary discharge. Concave plasma density profiles with axial electron density $>1 \times 10^{19} \mathrm{~cm}^{-3}$ were measured from discharge ablation of 330 or $440 \mu \mathrm{m}$ diameter $\mathrm{Ag}_{2} \mathrm{~S}$ capillaries with 3-5 kA peak amplitude current pulses. The dynamic of this plasma waveguide was studied with interferometry, absorption measurements, and hydrodynamic model simulations. The results are relevant to the development of efficient longitudinally pumped metal vapor soft x-ray lasers, in particular those employing transient excitation of Ni-like ions. An approach to the design of a gain saturated waveguided $13.9 \mathrm{~nm}$ laser in Ni-like Ag is discussed.
\end{abstract}

DOI: 10.1103/PhysRevE.72.026413

\section{INTRODUCTION}

The generation of dense plasma channels capable of extending the interaction length between intense laser pulses and plasmas beyond the limits imposed by diffraction and ionization-induced refraction is of significant interest for the development of efficient soft x-ray lasers and plasma accelerators. Several schemes for the generation of plasma waveguides based on either laser or discharge excitation have been studied [1-9]. Some of these plasma waveguides have been utilized in soft $\mathrm{x}$-ray laser amplification experiments [10-12]. Picosecond laser excitation of sulfur plasma columns created by an ablative capillary discharge has been demonstrated to produce lasing at $60.8 \mathrm{~nm}$ in Ne-like S [10]. Laser amplification by electron-ion recombination following optical-field ionization with intense ultrashort laser pulses has been reported at $13.5 \mathrm{~nm}$ within plasma channels created by discharge or laser ablation of $\mathrm{LiF}$ microcapillaries [11], and a collisionally excited optical-field ionization laser in Pd-like Xe at $41.8 \mathrm{~nm}$ has been recently reported using a plasma waveguide created in a gas-filled microcapillary excited by a relatively slow discharge current pulse [12]. The generation of elongated waveguides in highly ionized $\mathrm{Ar}$ plasmas created by a fast capillary discharge of the type used to develop discharge-pumped collisional soft $\mathrm{x}$-ray lasers has also been reported [9].

All of the above soft $\mathrm{x}$-ray laser amplification experiments have made use of plasmas created from either gases or relatively low $Z$ vapors. The development of efficient collisional soft $\mathrm{X}$-ray lasers operating below $20 \mathrm{~nm}$ can be achieved by the excitation of mid-to-high $Z \mathrm{Ni}$-like ions that with the exception of $\mathrm{Xe}$ are metals. Previous investigations of the ablation and excitation of evacuated low- $Z$ plastic microcapillaries by relatively low current pulses $(1.5 \mathrm{kA})$ revealed the formation of concave plasma density profiles with convex index of refraction that were demonstrated to be useful for guiding optical beams [4]. Herein we report the use of a fast $(\sim 55 \mathrm{~ns}$ first half-cycle) microcapillary discharge of larger peak current (3-6 kA) for the generation of dense plasma
PACS number(s): 52.38.Hb, 42.55.Vc, 52.38.Kd

waveguides containing a large concentration of silver ions. The plasma waveguides generated have electron densities that are suitable for the absorption of optical radiation and for the collisional excitation of soft x-ray laser transitions. The results are relevant to the development of a longitudinally pumped Ni-like Ag $(13.9 \mathrm{~nm})$ laser that takes advantage of transient collisional excitation to produce high gain coefficients $[13,14]$. The longitudinal excitation of such lasers is of significant interest as it can potentially result in saturated amplifiers with reduced laser pump energy requirements and increased efficiency.

\section{GENERATION AND CHARACTERIZATION OF THE CAPILLARY PLASMA}

The plasmas were generated by discharge ablation of the walls of $\mathrm{Ag}_{2} \mathrm{~S}$ capillaries 330 or $440 \mu \mathrm{m}$ in diameter and $2-4 \mathrm{~mm}$ in length. The capillary channels were constructed by drilling a hole into rods of $\mathrm{Ag}_{2} \mathrm{~S}$ created by pressing 99.998 percent pure $\mathrm{Ag}_{2} \mathrm{~S}$ powder at a pressure of 31 tons $\mathrm{cm}^{-2}$. The discharge current pulses, illustrated in Fig. 1 , had a peak amplitude of 3-5.5 kA and a half-period of $\sim 55 \mathrm{~ns}$. The current pulse was designed to be of short duration to help confine the plasma within the capillary channel, minimizing end-effects that could significantly degrade the guiding characteristics of the plasma column. To enhance the uniformity of the plasma column and its shot-to-shot reproducibility a preplasma was generated by filling the capillary channels with 0.6 Torr of $\mathrm{He}$ and preionizing the gas with a low current pulse.

The evolution of the plasma density distribution within the microcapillary was measured by interferometry at $267 \mathrm{~nm}$ using the third harmonic from a Ti:sapphire laser system producing $\sim 1 \mathrm{~mJ}, \sim 50 \mathrm{fs}$ laser pulses at $800 \mathrm{~nm}$. A conventional Mach-Zehnder interferometer was used for this purpose. Figure 2(a) shows a reference interferogram acquired prior to the initiation of the current pulse. Figure 2(b) shows an on-axis interferogram corresponding to a $440 \mu \mathrm{m}$ diameter $2 \mathrm{~mm}$ long $\mathrm{Ag}_{2} \mathrm{~S}$ capillary excited by a $5.2 \mathrm{kA}$ cur- 


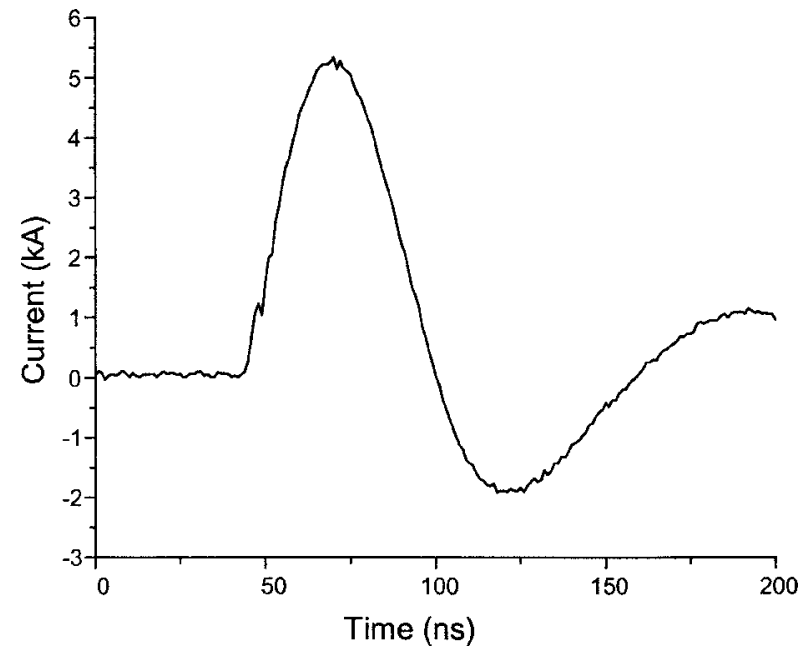

FIG. 1. Discharge current pulse used to excite a $440 \mu \mathrm{m}$ diameter, $2.2 \mathrm{~mm}$ long $\mathrm{Ag}_{2} \mathrm{~S}$ microcapillary plasma.

rent pulse, obtained $59 \mathrm{~ns}$ after the initiation of the current pulse. Figure 2(c) shows an overlay of an experimental interferogram and a simulated interferogram obtained adjusting an assumed electron density profile to best fit the experimental data. The electron density profile resulting from the fit is shown in Fig. 2(d). The discharge is observed to form a plasma channel with a minimum density on axis and steep walls, that is suitable for guiding laser light. The electron density difference between the axis of the channel and the vicinity of the walls is measured to be $\Delta N_{\mathrm{e}} \approx 1.0$ $\times 10^{19} \mathrm{~cm}^{-3}$. Figure 3 shows a sequence of interferograms that map the temporal evolution of the electron density distribution within the $440 \mu \mathrm{m}$ diameter $\mathrm{Ag}_{2} \mathrm{~S}$ capillary. It is observed that at these discharge conditions the time span during which the generation of concave electron density profile is observed ranges from about 50 to $70 \mathrm{~ns}$ from the beginning of the current pulse. For times longer than $\sim 70 \mathrm{~ns}$, after the end of the first half-cycle of the current, the concave density profile starts to degrade rapidly. It is of interest to notice that late in time, after a few half-periods of the current (e.g., 220 ns frame in Fig. 3), the curvature of the fringes reverses direction, indicating the presence of a convex electron density profile with maximum density on axis. This profile results from the fact that late in time, when the amplitude of the current has decreased significantly, the capillary walls are no longer a source of material, but a recombination site that constitutes a sink for charged particles.

It should be noted that the absolute value of the electron density on axis cannot be determined from the interferograms due to the difficulty of reliably determining the position of "zero density" fringes. This is due to the fact that nowhere within the capillary channel is the electron density zero, added to the fact that the position of the fringes in the reference interferograms obtained in the absence of a plasma [Fig. 2(a)] shifts from one laser shot to another introducing an additional complication. The electron density on axis was instead determined measuring the absorption of a $267 \mathrm{~nm}$ wavelength laser probe beam of $\sim 100 \mu \mathrm{m}$ diameter propagated along the capillary axis. Figure 4 shows the measured beam transmissivity as a function of time with respect to the
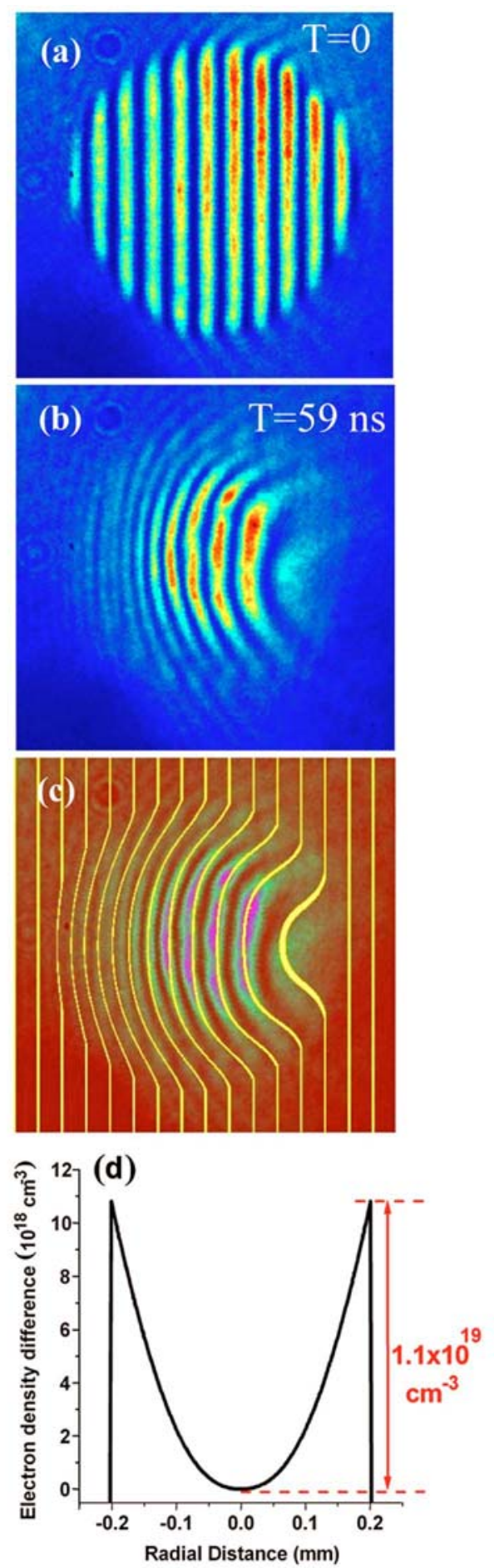

FIG. 2. (Color online) Interferogram of a $440 \mu \mathrm{m}$ diameter, $2 \mathrm{~mm}$ long $\mathrm{Ag}_{2} \mathrm{~S}$ capillary discharge plasma excited by a $5.2 \mathrm{kA}$ current pulse of $55 \mathrm{~ns}$ first half-cycle duration. The wavelength of the probe beam was $267 \mathrm{~nm}$. (a) Reference interferogram (no plasma present); (b) interferogram obtained 59 ns after the initiation of the current pulse; (c) overlay of measured and best-fit simulated interferogram; and (d) corresponding radial variation of the electron density distribution. Notice that due to the absence of reliable reference fringes the interferograms yield the radial variation of the electron density, but not the absolute value of the density. The onaxis electron density value was instead determined from absorption measurements. 

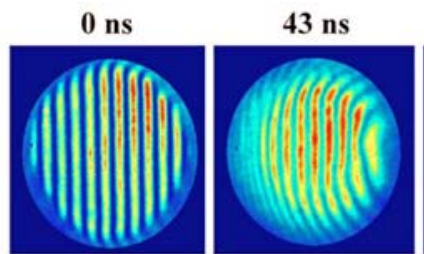

$51 \mathrm{~ns}$

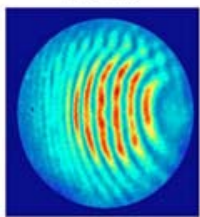

$58 \mathrm{~ns}$

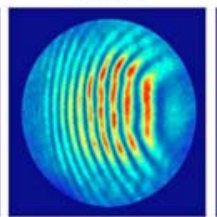

$72 \mathrm{~ns}$

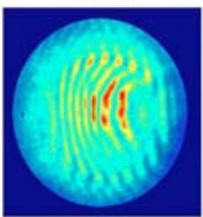

$81 \mathrm{~ns}$

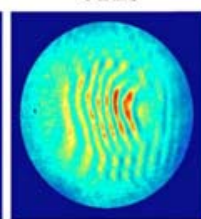

$220 \mathrm{~ns}$

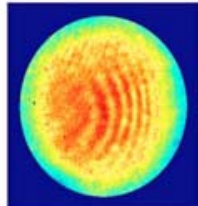

FIG. 3. (Color online) Sequence of interferograms corresponding to a $440 \mu \mathrm{m}$ diameter $\mathrm{Ag}_{2} \mathrm{~S}$ capillary. The times are measured with respect to the beginning of the current pulse.

origin of the current pulse for three different ranges of peak current pulse amplitudes. The electron density was computed from the absorption values using an electron temperature of $20-30 \mathrm{eV}$ and a mean ionization of $Z=7-8.9$ both estimated with the hydrodynamic model computations discussed below. The plasma column was assumed to be axially uniform, and end effects were neglected. Under these assumptions axial electron densities of $\sim(2-3) \times 10^{19} \mathrm{~cm}^{-3}$ were calculated from the measured absorptions for the majority of the shots within the time interval corresponding to the formations of strongly guiding density profiles ( $\sim 50-70 \mathrm{~ns})$. Discharges through smaller diameter capillaries were measured to generate plasmas with higher electron density on axis and steeper density walls. Interferograms from a $330 \mu \mathrm{m}$ diameter capillary excited by a $3.3 \mathrm{kA}$ current pulse $57 \mathrm{~ns}$ after the initiation of the current pulse show $\Delta N_{\mathrm{e}}=1.3$ $\times 10^{19} \mathrm{~cm}^{-3}$.

\section{MODELING OF THE DYNAMICS OF THE PLASMA}

The dynamics of the microcapillary plasma was modeled using the hydrodynamic/atomic code RADEX [15]. The simulations show that this type of discharge has many similarities with respect to the plastic microcapillary discharge plasmas we studied previously [4]. In both cases a low density

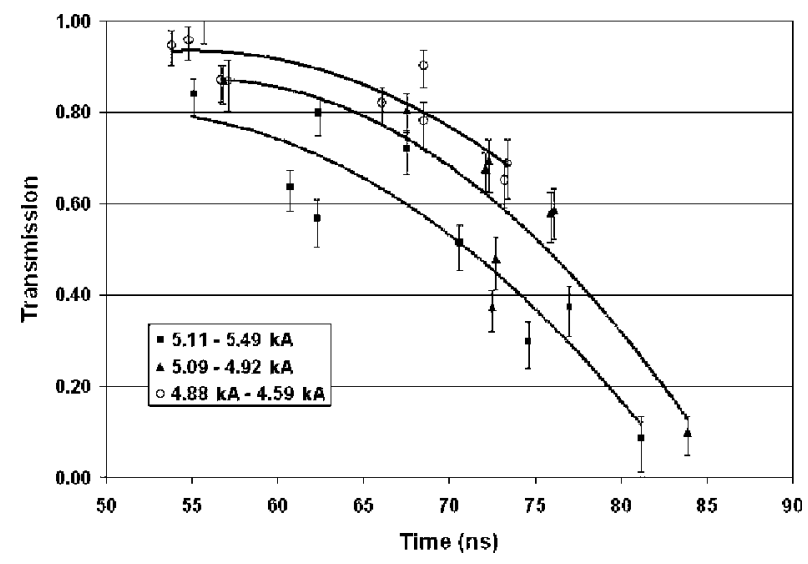

FIG. 4. Temporal variation of the transmissivity of a $267 \mathrm{~nm}$ beam through the axis of a $2.2 \mathrm{~mm}$ long $440 \mu \mathrm{m}$ diameter $\mathrm{Ag}_{2} \mathrm{~S}$ capillary plasma excited by a $55 \mathrm{~ns}$ half-period current pulse. Data for three different ranges of peak currents is shown. The error bars represent the uncertainty in determining the transmissivity of each individual shot. The scatter in the data illustrates the shot-to-shot variations. Variations in absorption were also observed from one capillary to another.
$10^{16}-10^{17} \mathrm{~cm}^{-3}$ precursor plasma is formed, which collapses with high speed $\left(\sim 10^{7} \mathrm{~cm} / \mathrm{s}\right)$ on axis reaching a high temperature and high degree of ionization. The plasma ablated from the capillary wall by the skin current rapidly moves towards the axis, filling the entire volume of the microcapillary. Because initially the mass of ablated material is low, the still small value of the rising current is capable of rapidly heating the plasma. As a result the plasma reaches a velocity in excess of $1 \times 10^{6} \mathrm{~cm} / \mathrm{s}$ in the first several $\mathrm{ns}$ of the discharge current pulse. Due to the fast process of plasma convection and diffusion the electromagnetic field embedded in the plasma flow quickly reaches the center of the capillary, resulting in maximum Joule heating near the capillary axis.

Figures 5(a) and 5(b) show the computed spatial-temporal variation of the electron temperature and the degree of ionization of the plasma of a $440 \mu \mathrm{m}$ diameter $\mathrm{Ag}_{2} \mathrm{~S}$ capillary excited by a $55 \mathrm{~ns}$ first half-cycle duration current pulse with a peak amplitude of $5.5 \mathrm{kA}$. The plasma quickly thermalizes during the first several ns. The $\mathrm{Ag}_{2} \mathrm{~S}$ material of the microcapillaries used in these experiments has a low sublimation/ activation energy threshold, similar to the plastics used in the previous experiments. The best agreement with experiment was obtained using an activation energy of the order of 0.2 to $0.3 \mathrm{eV}$. However, due to the higher temperature and the larger $Z$ of the increased discharge current experiments discussed herein (up to $\sim 50 \mathrm{eV}$ and up to 3 to 4 times higher maximum mean ionization $Z_{\max } \sim 13.5$ ), photorecombination and line radiation have a larger impact on wall ablation than in the case of the plasmas from low $\mathrm{Z}\left(\mathrm{CH}_{2} \mathrm{O}\right)_{n}$ materials with $Z_{\max } \sim 3-5$. The interferometric measurement shows that good guiding properties occur near the end of the first half-sine cycle of the current pulse. At this time a large amount of material has been ablated from the capillary wall and the shock waves on the electron density profile have relaxed, while the temperature and ion charge which peak earlier in the current pulse still keep substantial values: Te $\sim 20-30 \mathrm{eV}$ and total average ion charge $Z \sim 7-8.9$, with a mean charge of the Ag ion of $Z \sim 8.3-8.7$. During the second half-cycle of the current pulse, in which the current reaches only $\sim 0.5$ of the first half-cycle maximum value, the electron temperature does not increase beyond $15-20 \mathrm{eV}$, but is still enough to provide the inflow of new cold material into the capillary. It was computed that the electron density continues to grow beyond the value of $\sim 2 \times 10^{19} \mathrm{~cm}^{-3}$ reached near the end of the first half-cycle of the current. During this time the computed density profile (Fig. 6) has a clearly concave shape that forms due to pressure balance because of the obvious reason that the hotter plasma is generated near the axis of the discharge. The electron pressure substantially ex- 

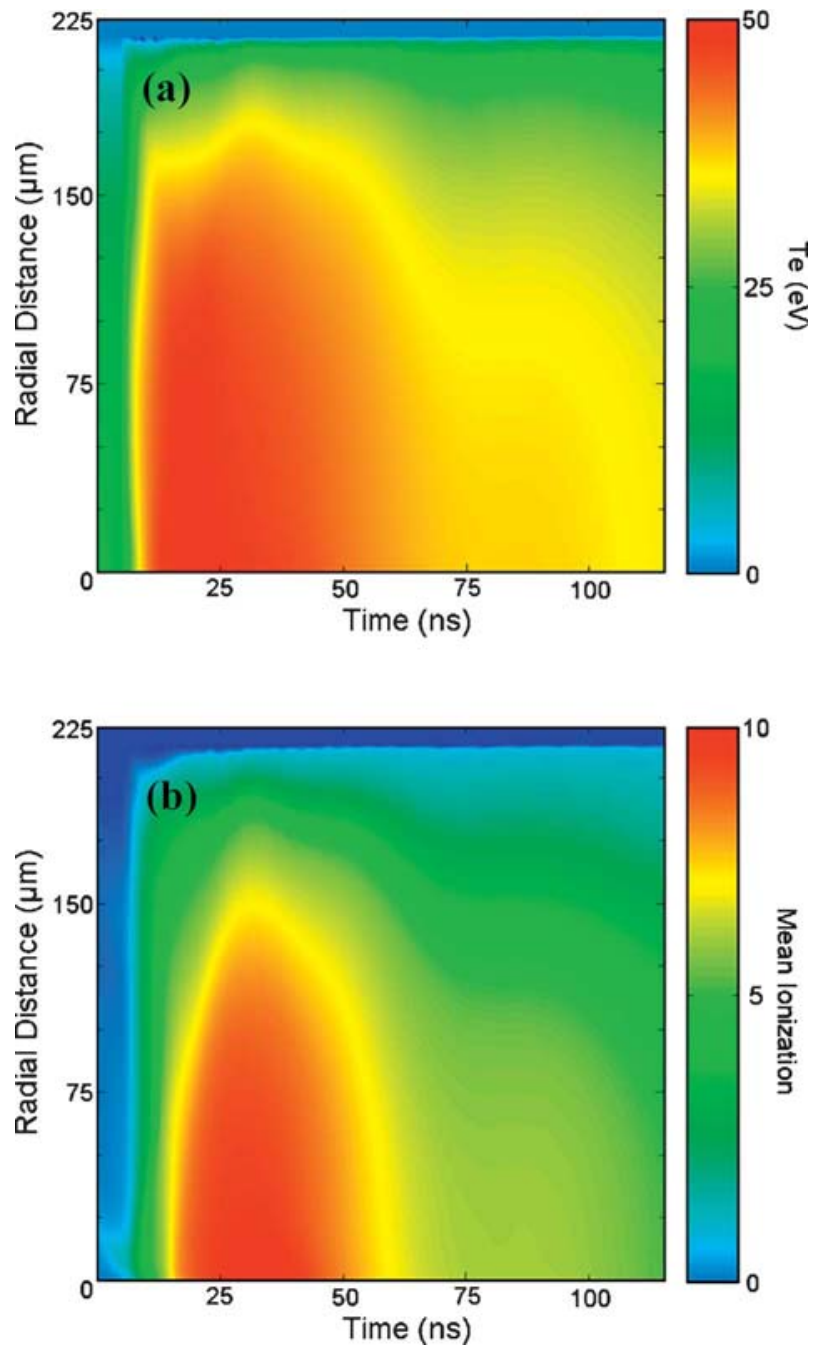

FIG. 5. (Color) (a) Computed evolution of the electron temperature in a $440 \mu \mathrm{m}$ diameter, $2 \mathrm{~mm}$ long $\mathrm{Ag}_{2} \mathrm{~S}$ capillary discharge plasma heated by a $5.2 \mathrm{kA}$ current pulse of $55 \mathrm{~ns}$ first half-cycle duration. (b) Corresponding computed variation of the mean degree of ionization $Z$.

ceeds the magnetic and ion pressures at this time.

The RADEX calculations showed that despite the use of third harmonic light the probe beam is substantially refracted for microcapillary lengths $>4 \mathrm{~mm}$. Therefore the interferometry and absorption measurements were obtained using

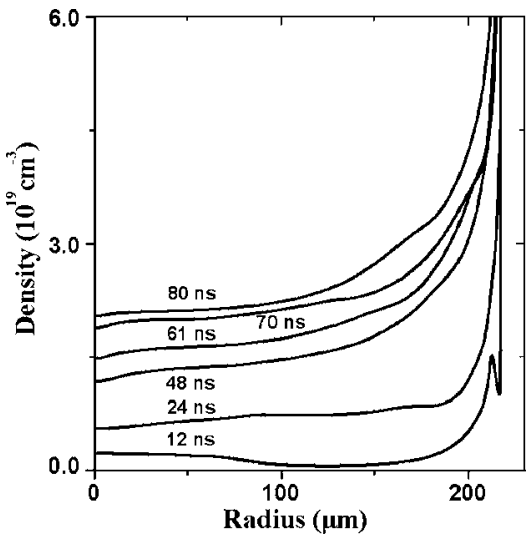

FIG. 6. Sequence of computed electron density profiles corresponding to a $440 \mu \mathrm{m}$ diameter $\mathrm{Ag}_{2} \mathrm{~S}$ microcapillary driven by $5.5 \mathrm{kA}$ current pulse of $55 \mathrm{~ns}$ first half-cycle duration. The labels indicate the time respect to the beginning of the current pulse

2-2.2 mm long capillaries. The computed electron density profiles of Fig. 6 reproduce well the electron distributions measured with interferometry, and corroborates the guiding characteristics of these discharges. Also the on-axis density values are in relatively good agreement with the experimentally measured absorption of the $267 \mathrm{~nm}$ probe beam, though at later times $(>60 \mathrm{~ns})$ the modeled densities are somewhat lower than experimental estimations based on transmission data. For $330 \mu \mathrm{m}$ diameter capillaries the electron density on axis is computed to reach $4.4 \times 10^{19} \mathrm{~cm}^{-3}$, with approximately the same electron temperature and degree of ionization.

\section{GUIDING CHARACTERISTICS}

Modeling of the guiding characteristics of these plasmas was accomplished by first determining a radially dependent phase delay based on the interferometrically measured electron density profiles, and subsequently propagating the beam using a fast-Fourier transform algorithm. The measured electron density profile for a $440 \mu \mathrm{m}$ diameter capillary shown in Fig. 2 is computed to guide an $800 \mathrm{~nm}$ wavelength beam with a matched mode size of $\omega_{0}=25 \mu \mathrm{m}$, as illustrated in Fig. 7.

Beam propagation experiments were conducted with laser pulses generated from a mode-locked Ti:sapphire oscillator,stretched to $\sim 120 \mathrm{ps}$ and subsequently amplified to

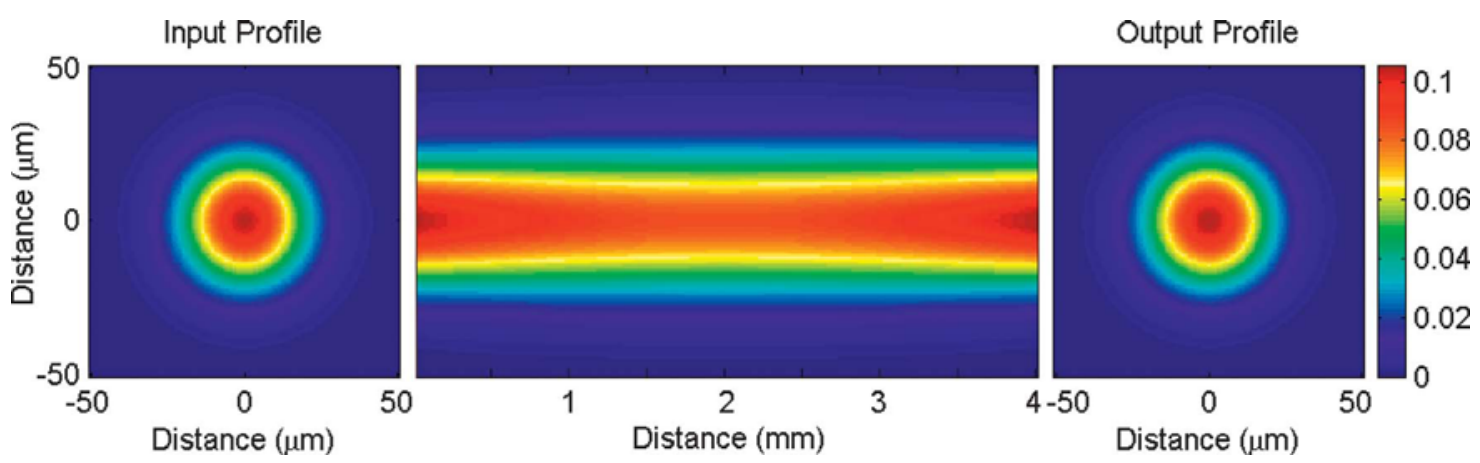

FIG. 7. (Color) Simulated propagation of a $800 \mathrm{~nm}$ wavelength laser beam through a plasma channel with the measured electron density profile shown in Fig. 2(d). To more clearly show the guiding effect the attenuation of the beam due to absorption was neglected. 

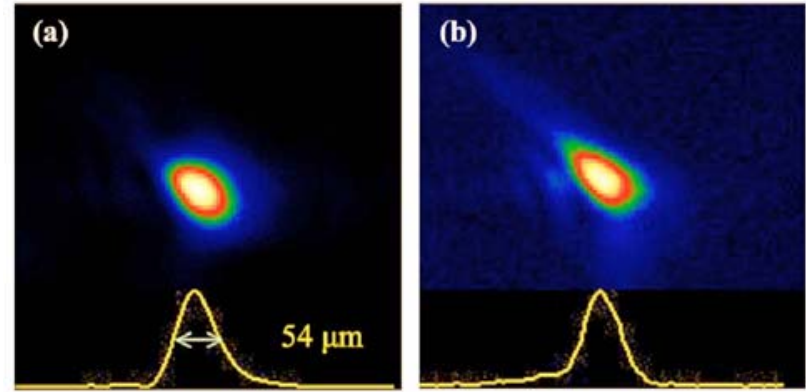

FIG. 8. (Color online) Exit beam intensity distribution patterns corresponding to a Ti:sapphire laser beam propagated through a $440 \mu \mathrm{m}$ diameter, $4 \mathrm{~mm}$ long $\mathrm{Ag}_{2} \mathrm{~S}$ capillary. The laser pulse had an energy of $100 \mathrm{~mJ}$ and a pulse duration of $120 \mathrm{ps}$.

$\sim 100 \mathrm{~mJ}$ in a two-stage amplifier system. The pulses were focused into a $2 \omega_{0} \sim 60 \mu \mathrm{m}$ spot at the entrance of the capillary channel. The output plane of the capillary was imaged onto a charge-coupled device camera. Figure 8 shows the exit mode patterns corresponding to a $440 \mu \mathrm{m}$ diameter, $4 \mathrm{~mm}$ long capillary, acquired $55 \mathrm{~ns}$ after the initiation of the current pulse. Figure 8(a) shows an output mode pattern with a well-defined peak of $\sim 54 \mu \mathrm{m}$ full width at half maximum and a relatively low pedestal. However, we also observed shot-to-shot variations in the exit beam intensity distribution, with some shots showing a significantly more spread exit beam distribution such as that in Fig. 8(b).

\section{APPROACH TO A 13.9 nm Ni-LIKE Ag LASER}

The waveguiding properties of microcapillary could be beneficial for the development of compact x-ray lasers. The lasing scheme could vary, but these metal vapor plasma waveguides are directly applicable to $\mathrm{x}$-ray lasers based on the transient collisional excitation approach [13-15]. The creation of the concave plasma density profile makes it possible to guide the heating laser pulse and create the plasma parameters needed for x-ray laser amplification over distances exceeding many Rayleigh lengths. Initially, the capillary discharge plasma is not sufficiently ionized for lasing at $13.9 \mathrm{~nm}$ in Ni-like $\mathrm{Ag}(\mathrm{Ag} \mathrm{XX})$ by inversion of the $4 d^{1} S_{0}$ $-4 p^{1} P_{1}$ transition. Additional plasma heating and ionization must be therefore generated by externally injected laser light. When the large laser power is deposited in neutral gases or plasmas with a low degree of ionization and substantial density (and for shorter wavelength $\mathrm{x}$-ray lasers it is important to operate at large plasma densities to compensate for the rapid decrease of the gain with wavelength) ionization-induced defocusing limits the length of the plasma. In our case, the plasma is initially already substantially ionized, therefore the ion charge has to increase not an order of magnitude but just about a factor of 2 . There exist several possible ways to create the additional ionization and heating necessary to create the population inversion required for lasing. Using short laser pulses of high intensity produced by a chirped-pulseamplification laser it is possible to further ionize and heat the plasma. This can be achieved either via optical field ionization $[16,17]$ applying femtosecond-scale laser pulses of circularly polarized radiation with an intensity of more than
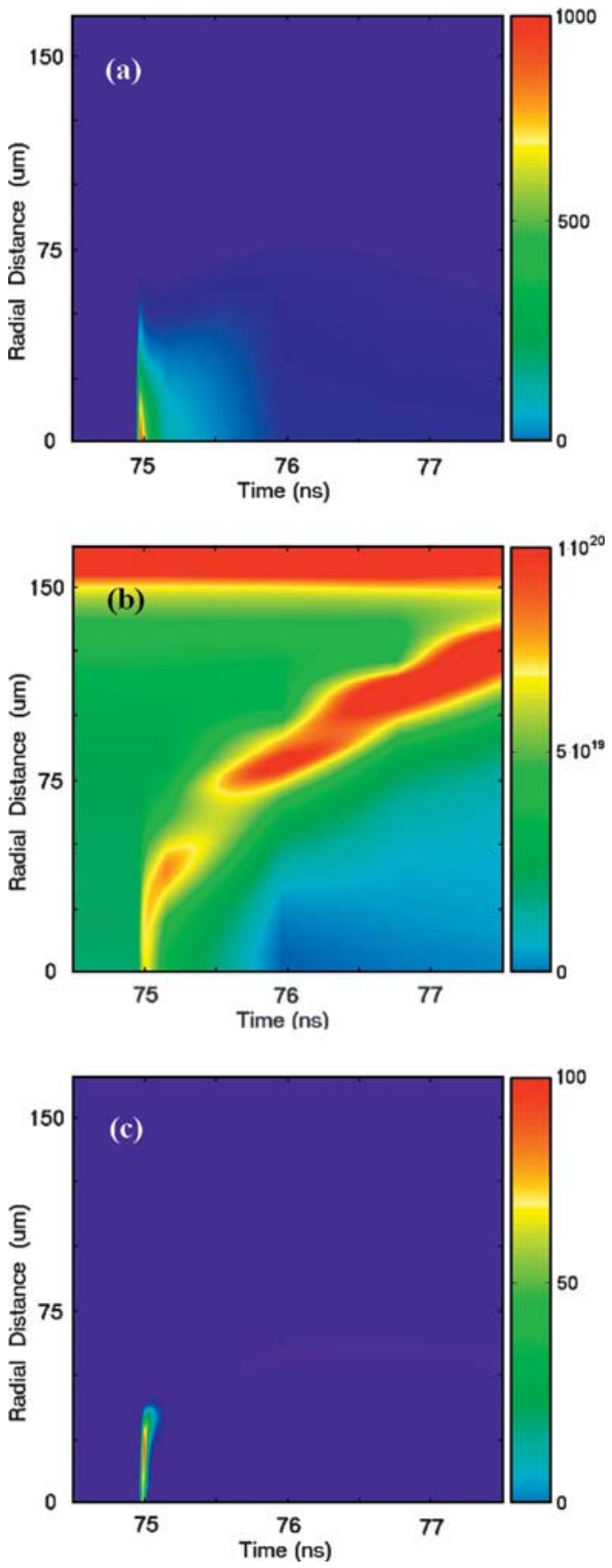

FIG. 9. (Color) Computed plasma parameters and gain in the $13.9 \mathrm{~nm}$ line of Ni-like Ag following the rapid heating of the capillary plasma with a $10 \mathrm{ps}$ duration $\lambda=1.06 \mu \mathrm{m}$ laser pulse with an intensity of $1.5 \times 10^{15} \mathrm{~W} / \mathrm{cm}^{2}$. (a) Calculated evolutions of electron temperature $(\mathrm{eV}),(\mathrm{b})$ electron density restricted to $1 \times 10^{20}\left(\mathrm{~cm}^{-3}\right)$, and (c) gain coefficient for the $13.9 \mathrm{~nm}$ line of $\mathrm{Ag} \mathrm{Xx}\left(\mathrm{cm}^{-1}\right)$. The laser pulse is injected $75 \mathrm{~ns}$ from the beginning of the current pulse. The microcapillary diameter is $330 \mu \mathrm{m}$.

$10^{17} \mathrm{~W} / \mathrm{cm}^{2}$, or by inverse-bremsstrahlung absorption with more moderate fluxes $(3-15) \times 10^{14} \mathrm{~W} / \mathrm{cm}^{2}$ of approximately the same energy and longer picosecond-scale pulse durations. Below we discuss the later case, supported by model computations performed with the code RADEX.

For these calculations we assumed the use of the smaller bore capillaries with a diameter of $330 \mu \mathrm{m}$, which produce a 
larger plasma density that in turn results in an increased pump laser absorption efficiency over a selected length of $1 \mathrm{~cm}$ of the medium. The short laser heating pulse of 5-30 ps duration must be deposited at 50-70 ns after the initiation of the current pulse, time at which the capillary discharge plasma column presents a concave guiding density profile. The increased plasma heating leads to larger final density and gain. We conducted simulations for a $\lambda$ $=1.06 \mu \mathrm{m}$ laser heating pulse of $10 \mathrm{ps}$ duration with an intensity of $1.5 \times 10^{15} \mathrm{~W} / \mathrm{cm}^{2}$. During the pump laser pulse the temperature reaches $\sim 1 \mathrm{keV}$ [see Fig. 9(a)] which is sufficient to ionize the plasma to $\mathrm{Ag} \mathrm{XX}$ (potential of ionization $\sim 890 \mathrm{eV}$ ) at the end of the pulse. The electron density jumps from an initial value of $3.5 \times 10^{19} \mathrm{~cm}^{-3}$ to $8 \times 10^{19} \mathrm{~cm}^{-3}$ Since the laser pulse is sufficiently short, the plasma ionizes without a significant hydrodynamic expansion [see the density peak at $75 \mathrm{~ns}$ in Fig. 9(b)], keeping the concave profile necessary to maintain the waveguiding properties. Subsequently the large plasma pressure and its gradient produce a strong shock wave moving outward from the capillary axis, which after $0.5-1 \mathrm{~ns}$ leads to a decrease of the axial density, forming there a large cavity. For a $0.5-1 \mathrm{~cm}$ long plasma column with a density of $(6-8) \times 10^{19} \mathrm{~cm}^{-3}$ and an electron temperature of $800-1000 \mathrm{eV}$ the absorption coefficient due to inverse bremsstrahlung reaches $1 \mathrm{~cm}^{-1}$ ensuring high absorption efficiency of $\sim 50 \%$ together with a good plasma homogeneity.

The gain in the $4 d^{1} S_{0}-4 p^{1} P_{1}$ transition is predicted to reach substantial values $\sim 50-100 \mathrm{~cm}^{-1}$ at the time corresponding to the end of the heating pulse. The $\mathrm{x}$-ray laser amplification process at $13.9 \mathrm{~nm}$ will also benefit from the minimization of refraction that results from the waveguiding properties of the amplification medium. With these gain coefficient values gain saturation would be achieved for plasma column lengths of only a few millimeters, making it possible to operate a $1 \mathrm{~cm}$ long amplifier in the highly saturated regime as necessary for efficient laser energy extraction. The gain is computed to have a duration of $10-20$ ps [Fig. 9(c)], and the soft $\mathrm{x}$-ray laser output pulse energy can be expected to be of the order of $10 \mu \mathrm{J}$. According to the model computations these results could be achieved with a laser pump energy of 300-500 mJ, that is smaller than that used to produce saturated lasing in Ni-like $\mathrm{Ag}$ by both transverse $[14,18]$ or grazing incidence pumping [19], due to the higher absorption of the pump radiation.

\section{CONCLUSIONS}

In summary, we have demonstrated the generation of plasma waveguides containing a large density of $\mathrm{Ag}$ ions and axial electron densities of $(2-3) \times 10^{19} \mathrm{~cm}^{-3}$. This type of capillary plasma waveguides containing mid- $Z$ ions is of interest for the development of axially excited collisional soft $\mathrm{x}$-ray lasers at wavelengths below $20 \mathrm{~nm}$.

\section{ACKNOWLEDGMENTS}

This work was supported by the U.S. Department of Energy, Chemical Science, Geosciences and Biosciences Division of the office of Basic Energy Sciences, and NSF Center for Extreme Ultraviolet Science and Technology under NSF Award Number EEC-0310717 with equipment developed under NSF Grant No. ECS-9977677. We also gratefully acknowledge the support of the W. M. Keck Foundation.
[1] C. G. Durfee III and H. M. Milchberg, Phys. Rev. Lett. 71, 2409 (1993); C. G. Durfee III, J. Lynch, and H. M. Milchberg, Phys. Rev. E 51, 2368 (1995).

[2] Y. Ehrlich, C. Cohen, A. Zigler, J. Krall, P. Sprangle, and E. Esarey, Phys. Rev. Lett. 77, 4186 (1996).

[3] J. J. Rocca, F. G. Tomasel, J. L. A. Chilla, C. H. Moreno, B. R. Benware, V. N. Shlyaptsev, J. J. Gonzalez, and C. D. Macchietto, Proc. SPIE 3156, 164 (1997).

[4] M. C. Marconi, C. H. Moreno, J. J. Rocca, V. N. Shlyaptsev, and A. L. Osterheld, Phys. Rev. E 62, 7209 (2000).

[5] S. M. Hooker, D. J. Spence, and R. A. Smith, J. Opt. Soc. Am. B 17, 90 (2000).

[6] A. Butler, D. J. Spence, and S. M. Hooker, Phys. Rev. Lett. 89, 185003 (2002).

[7] T. Hosakai, M. Kando, H. Dewa, H. Kotaki, S. Kondo, N. Hasagawa, K. Nakajima, and K. Horioka, Opt. Lett. 25, 10 (2000)

[8] C. Fauser and H. Langhoff, Appl. Phys. B: Lasers Opt. B71, 607 (2000).

[9] B. M. Luther, Y. Wang, M. C. Marconi, J. L. A. Chilla, M. A. Larotonda, and J. J. Rocca, Phys. Rev. Lett. 92, 235002 (2004)

[10] K. A. Janulewicz, J. J. Rocca, F. Bortolotto, M. P. Kalachnikov, V. N. Shlyaptsev, W. Sandner, and P. V. Nickles, Phys. Rev. A 63, 033803 (2001).
[11] D. V. Korobkin, C. H. Nam, S. Suckewer, and A. Goltsov, Phys. Rev. Lett. 77, 5206 (1996).

[12] A. Butler, A. J. Gonsalves, C. M. McKenna, D. J. Spence, S. M. Hooker, S. Sebban, T. Mocek, I. Bettaibi, and B. Cros, Phys. Rev. Lett. 91, 205001 (2003).

[13] P. V. Nickles, V. N. Shlyaptsev, M. P. Kalachnikov, M. Schnurer, I. Will, and W. Sandner, Phys. Rev. Lett. 78, 2748 (1997).

[14] J. Dunn, Y. Li, A. L. Osterheld, J. Nilsen, J. R. Hunter, and V. N. Shlyaptsev, Phys. Rev. Lett. 84, 4834 (2000).

[15] A. V. Vinogradov and V. N. Shlyaptsev, Sov. J. Quantum Electron. 13, 298 (1983); 13, 1511 (1983); Yu. V. Afanasiev et al., Sov. J. Laser Res. 10, 1 (1989); V. N. Shlyaptsev, J. J. Rocca, and A. L. Osterheld, Proc. SPIE 2520, 365 (1995).

[16] B. E. Lemoff, G. Y. Yin, C. L. Gordon III, C. P. J. Barty, and S. E. Harris, Phys. Rev. Lett. 74, 1574 (1995).

[17] S. Sebban, R. Haroutunian, P. Balcou, G. Grillon, A. Rousse, S. Kazamias, T. Marin, J. P. Rousseau, L. Notebaert, M. Pittman, J. P. Chambaret, A. Antonetti, D. Hulin, D. Ros, A. Klisnick, A. Carillon, P. Jaegle, G. Jamelot, and J. F. Wyart, Phys. Rev. Lett. 86, 3004 (2001).

[18] K. A. Janulewicz, A. Lucianetti, G. Priebe, W. Sandner, and P. V. Nickles, Phys. Rev. A 68, 051802(R) (2003).

[19] Y. Wang, M. A. Larotonda, B. M. Luther, D. Alessi, M. Berrill, J. J. Rocca, and V. N. Shlyaptsev, Phys. Rev. A (to be published). 